University of Nebraska - Lincoln

DigitalCommons@University of Nebraska - Lincoln

\title{
Negative-ion resonances in zinc: A study in elastic electron scattering and electron transmission
}

\author{
S. A. Napier \\ University of Western Australia \\ D. Cvejanović \\ University of Western Australia \\ Paul D. Burrow \\ University of Nebraska-Lincoln, pburrow1@unl.edu \\ J. F. Williams \\ University of Western Australia \\ J. A. Michejda \\ University of Nebraska-Lincoln \\ See next page for additional authors
}

Follow this and additional works at: https://digitalcommons.unl.edu/physicsburrow

Part of the Physics Commons

Napier, S. A.; Cvejanović, D.; Burrow, Paul D.; Williams, J. F.; Michejda, J. A.; and Pravica, L., "Negative-ion resonances in zinc: A study in elastic electron scattering and electron transmission" (2009). Paul Burrow Publications. 34.

https://digitalcommons.unl.edu/physicsburrow/34

This Article is brought to you for free and open access by the Research Papers in Physics and Astronomy at DigitalCommons@University of Nebraska - Lincoln. It has been accepted for inclusion in Paul Burrow Publications by an authorized administrator of DigitalCommons@University of Nebraska - Lincoln. 


\section{Authors}

S. A. Napier, D. Cvejanović, Paul D. Burrow, J. F. Williams, J. A. Michejda, and L. Pravica 


\title{
Negative-ion resonances in zinc: A study in elastic electron scattering and electron transmission
}

\author{
S. A. Napier, ${ }^{1}$ D. Cvejanović, ${ }^{1,2}$ P. D. Burrow, ${ }^{3}$ J. F. Williams, ${ }^{1,2, *}$ J. A. Michejda, ${ }^{3}$ and L. Pravica ${ }^{1,2}$ \\ ${ }^{1}$ Centre for Atomic, Molecular and Surface Physics, School of Physics, University of Western Australia, Perth 6009, Australia \\ ${ }^{2}$ ARC Centre for Antimatter-Matter Studies, School of Physics, University of Western Australia, Perth 6009, Australia \\ ${ }^{3}$ Department of Physics and Astronomy, University of Nebraska, Lincoln, Nebraska 68588-0111, USA
}

(Received 12 August 2009; published 22 October 2009)

\begin{abstract}
The energies, widths, and angular behavior of negative-ion resonances in zinc atoms are determined using the complementary experimental techniques of electron transmission and angular differential elastic scattering. The observed resonances include states formed by the excitation of an inner $3 d$ electron and assigned to a $3 d^{9} 4 s^{2} n \ell^{2}$ electron configuration, $\ell=1$ for $n=4$ and $\ell=0,1$ for $n=5$. The differential elastic scattering indicates that all negative ions in the $3 d^{9} 4 s^{2} 4 p^{2}$ manifold are formed from, and decay by, emission of a $d$-wave electron.
\end{abstract}

DOI: $10.1103 /$ PhysRevA.80.042710

PACS number(s): $34.80 . \mathrm{Dp}$

\section{INTRODUCTION}

The characteristics of short-lived negative-ion states have been studied extensively by electron scattering from atoms and have provided information about electron correlations in atoms [1]. Our recent study using spin polarized electrons illustrated [2] in a relatively light atom, zinc $(Z=30)$, how the spin-orbit interaction can be observed. In the present study, the techniques of electron transmission and angular differential elastic scattering are used in the vicinity of the excitation thresholds of the neutral and autoionizing thresholds from 3.5 to $16.7 \mathrm{eV}$ to characterize numerous negativeion resonances.

The few previous observations of negative ions in zinc include transmission [3,4], photon emission [2,5-7] and one differential elastic [4] and excitation ${ }^{3} P_{0,1,2}[8]$ observation. Also one calculation using the B-spline R-matrix model (BSRM) [9] covered the resonance region up to about $8 \mathrm{eV}$ in good agreement with experiments.

The existing data on negative-ion resonances in the autoionizing region of zinc $[4,6,7,9]$ show a wealth of resonance structure, however, the interpretations of results from different experiments and different scattering processes are generally inconclusive and identification of angular momentum coupling and associated spin effects are lacking. The important effects seem to originate from specific properties of the $3 d$ orbital and depend on the balance of magnetic and electrostatic interactions in the atom during the excitation of a $3 d$ electron.

To make further advances we used the transmission technique [10] with high sensitivity and high energy resolution to resolve the multiplet structures and to measure higher resonance series. In transmission, resonances are observed in the total scattering cross section and with high sensitivity by measuring the derivative of transmitted current with respect to energy. The high data collection efficiency permits spectra with high energy resolution that are less affected by the energy drifts sometimes associated with metal vapors. The angle differential elastic electron scattering, while benefiting from a reasonably large cross section, detects a well defined

\footnotetext{
*jfw@cyllene.uwa.edu.au
}

scattering event where initial and final target states are the same. In the case of an isolated resonance, the analysis of the angle differential elastic scattering can give information on the dominant partial wave taking part in both the resonance formation and decay leading toward assignment of the resonance symmetry. Using the two techniques, new spectroscopic information on the temporary negative ions in the autoionizing region of zinc has been obtained for the $3 d^{9} 4 s^{2} 4 p^{2}, 3 d^{9} 4 s^{2} 5 s^{2}$, and $3 d^{9} 4 s^{2} 5 p^{2}$ states.

\section{EXPERIMENT}

Elastic scattering was studied using a low energy, electron impact spectrometer for the incident and scattered electrons. The essential details are outlined here. Electrons emitted from a hairpin cathode are focused into a $127^{\circ}$ cylindrical energy selector and then by a system of three element aperture lenses into the interaction region where they cross the atomic beam. The zinc atoms are produced in a thermally heated molybdenum oven and emanate from a $15 \mathrm{~mm}$ long, 1 $\mathrm{mm}$ inner diameter tube. The electrons scattered into a given solid angle are energy analyzed and detected by a Photonis BL719 channel electron multiplier. The scattered electron energy analysis was performed using a $127^{\circ}$ cylindrical analyzer, which could rotate in an angular range from $\theta=0^{\circ}$ to $110^{\circ}$. For a fixed energy loss (zero for elastic scattering) the scattered electron intensity is measured as a function of energy by scanning together the incident energy and the detected electron energy. In this way, the shape of the differential cross section is determined as a function of energy.

The typical electron energy resolution varied from 90 to $110 \mathrm{meV}$. The calibration of the electron energy was performed by scanning the incident electron energy through the $4 s 4 d^{1} D_{2}$ state threshold while detecting photons emitted from this state in a direction perpendicular to the scattering plane. A step-like shape of the cross section at the threshold of this state was fitted to the theoretical cross section of Zatsarinny and Bartschat [9] convoluted with the Gaussian apparatus function corresponding to the particular experiment. This procedure resulted in an energy calibration uncertainty of $30 \mathrm{meV}$.

An instrumental background for all measured data was estimated by measuring the energy loss spectrum at several 

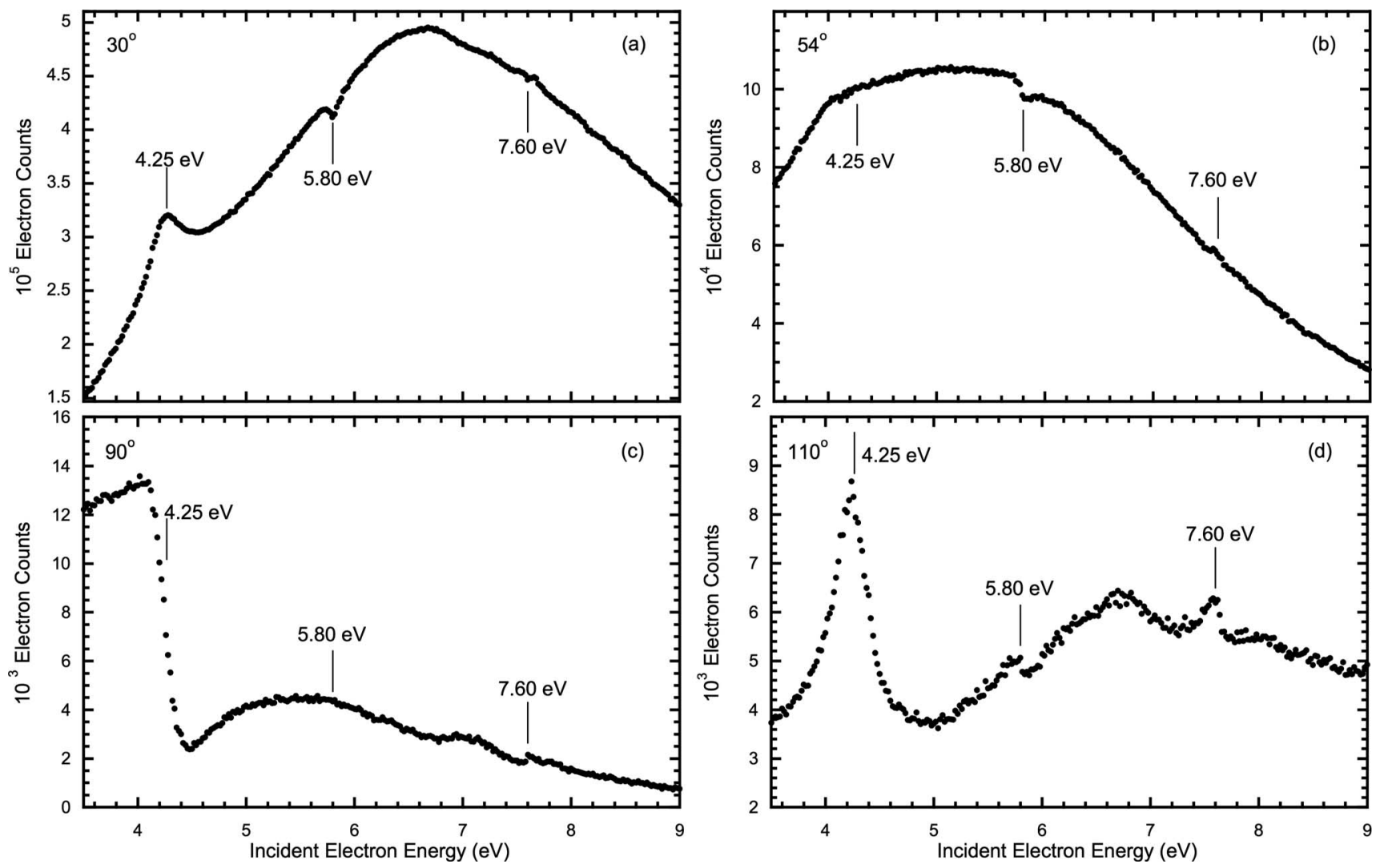

FIG. 1. Differential elastic scattering signal at a scattering angle of (a) $30^{\circ}$, (b) $54^{\circ}$, (c) $90^{\circ}$, and (d) $110^{\circ}$. The features near $4.25,5.80$, and $7.60 \mathrm{eV}$ are shown in greater detail in Figs. 2-4.

energies, then interpolating throughout the energy range and subtracting from the measured spectrum. Also an estimate of the elastic scattering from the residual gas was made by performing measurements with the exit tube of the oven blocked by a metal plate controlled from outside the vacuum. Therefore the percentage resonance contributions given in this paper give the true values relative to nonresonance scattering.

The electron transmission spectrometer used in these studies is in the format devised by Sanche and Schulz [10], and its previous application to the study of resonances in alkali metals is described in more detail elsewhere [11]. Briefly, the apparatus employs a magnetically collimated trochoidal electron monochromator [12] to generate an electron beam that is passed through a heated gas cell. A separate temperature controlled reservoir containing zinc metal is attached to the collision cell. The cell is kept approximately $100{ }^{\circ} \mathrm{C}$ hotter to minimize condensation and contact potential drifts. A metal cylinder inside the cell is used to modulate the electron energy and a phase-sensitive detector measures the energy derivative of the transmitted electron current. Electrodes between the collision cell and the electron beam collector are biased to discriminate against scattered electrons. Energy resolution is typically $30-50 \mathrm{meV}$. The energy scale is calibrated to within $\pm 0.030 \mathrm{eV}$ by reference to sharp vibrational structure near $2 \mathrm{eV}$ in the transmission spectrum of $\mathrm{N}_{2}$.

\section{RESULTS}

Negative-ion resonances in zinc were studied in a broad energy range using the techniques of differential elastic scat- tering from 3.5 to $13 \mathrm{eV}$ and electron transmission up to 17 $\mathrm{eV}$. The intensity of elastically scattered electrons was measured at four scattering angles $\theta=30^{\circ}, 54^{\circ}, 90^{\circ}$, and $110^{\circ}$. The results below the ionization threshold are shown in Fig. 1 , with details of the observed features and their numerical analysis shown in Figs. 2-4. The resonance contribution as a percentage of the interpolated (Figs. 2 and 3) or estimated (Fig. 4) nonresonance background is also shown. For the energy range from 3.5 to $9 \mathrm{eV}$, shown in Fig. 1, the spectrometer was not optimized always for constant transmission with the result that the measured intensities for elastic scattering are not necessarily accurate indications of the energy dependence of the cross sections. For the energy scans above $10 \mathrm{eV}$ and over a range of a few $\mathrm{eV}$ encompassing the resonance shapes, the variation in transmission of the electron optics was small. Above the ionization threshold, the results of the present transmission studies are shown in Figs. 6, 7, and $8(\mathrm{~b})$ and results for differential elastic scattering are shown in Figs. 8(a) and 9.

The transmission data, shown in Figs. 5 and 8(b), have been manipulated to permit a more direct comparison with the elastic scattering measurements. First, because they are differential with respect to energy, the transmission data were integrated. Second, the resonance contributions to the integrated transmission signal were isolated by subtracting a least-squares polynomial fit to the slowly varying nonresonance background. Last, the data were inverted because an increase (decrease) in the transmitted current corresponded to a decrease (increase) in the scattering signal. The effects observed in the transmission data are summed over all scat- 

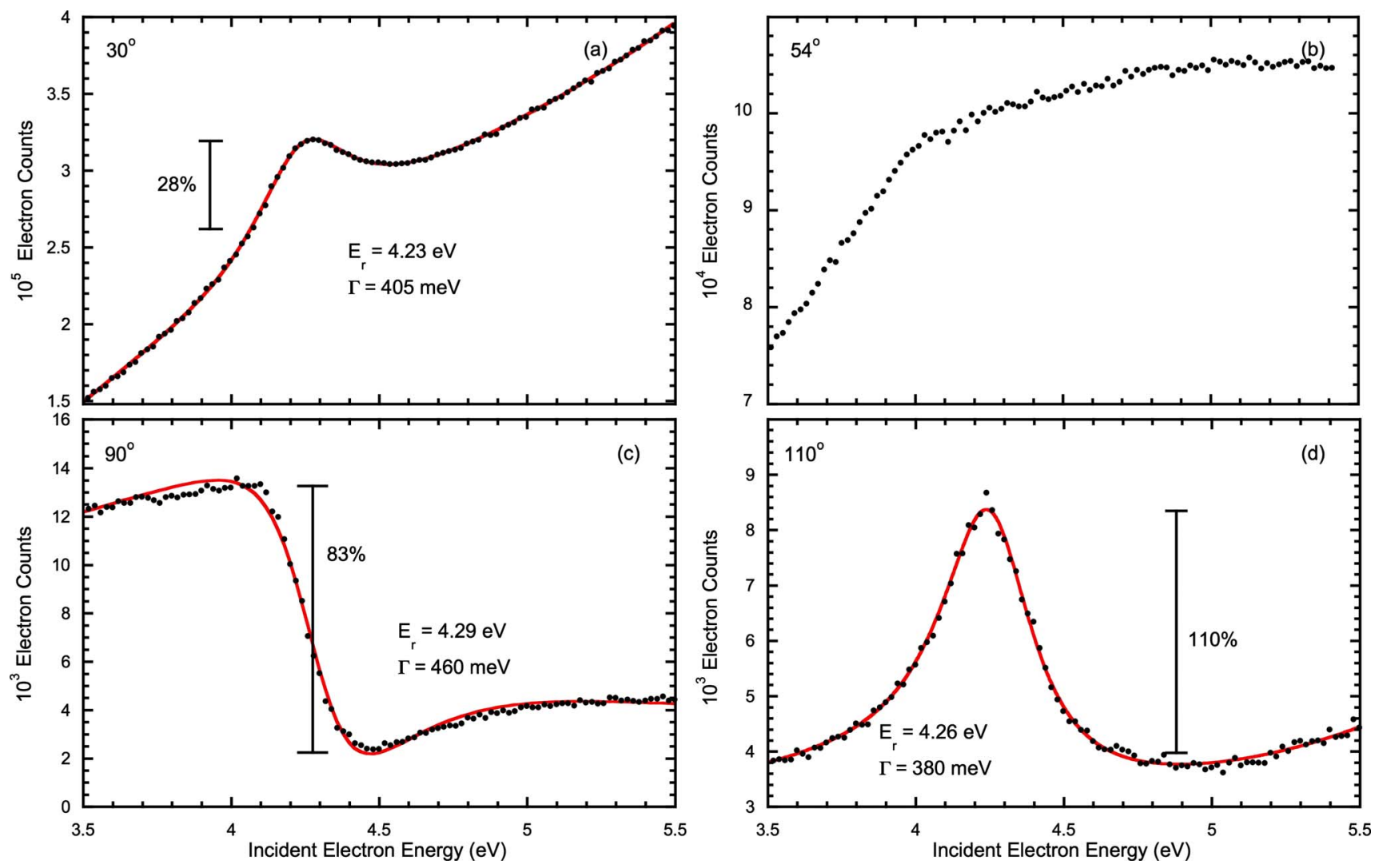

FIG. 2. (Color online) Differential elastic scattering signal in the vicinity of the $4.25 \mathrm{eV}$ negative ion resonance at a scattering angle of (a) $30^{\circ}$, (b) $54^{\circ}$, (c) $90^{\circ}$, and (d) $110^{\circ}$. present data, a combined polynomial background and Fano profile fit to the resonance structure, which obtained the resonance energy and width displayed. The size of the resonance, where present, is also indicated as a percentage of the nonresonance background.

tering channels, but one would expect small angle elastic scattering to dominate since that signal, as evidenced by an energy-loss spectrum at a scattering angle of $15^{\circ}$, was by far the most significant in this energy region. Accordingly, in Fig. 8 the integrated and inverted transmission data are placed next to the $15^{\circ}$ elastic differential scattering signal near $11 \mathrm{eV}$ to permit a direct comparison of resonance structures.

The experimental data from the differential elastic electron scattering were analyzed in two different ways depending on the separation of the individual resonance features. For well-separated resonance features the fitted function was a combination of a polynomial background and Fano profile with a least-squares fitting method to obtain resonance energies and a convoluted width. The fitting procedure has been given in detail in [6].

In the case of closely spaced and overlapping features mainly in the elastic scattering above the ionization threshold, the negative-ion resonances were isolated by subtracting a least-squares polynomial fit to the slowly varying background contribution. This estimated background is shown as a solid line (for example in Figs. 8 and 9) along with the isolated structure contributions as a percentage of the interpolated background. By subtracting a background, the relative size of the various resonance structures, as well as details of their shape, become more apparent. In this case, the resonance energies were determined from a peak fit to the extreme parts of each feature.
The total energy uncertainty for any of the resonances quoted in the present paper includes uncertainty from the fitting procedure, the calibration uncertainty and uncertainty due to energy drifts.

\section{A. Below the ionization threshold}

Differential elastic scattering signals at scattering angles of $30^{\circ}, 54^{\circ}, 90^{\circ}$, and $110^{\circ}$ in Fig. 1 show two well-isolated features at a mean energy of 4.25 and $5.80 \mathrm{eV}$. The details of their angular behavior are shown in Figs. 2 and 3, respectively. The energies and apparent widths of the structures, as obtained from the of numerical fitting to Fano profiles at each scattering angle where a resonance is observable, are shown in Figs. 2 and 3 and also in Table I.

From Fig. 2 it is seen that the resonance at $4.25 \mathrm{eV}$ is not visible at $54^{\circ}$, which indicates a $d$-wave nature. Very strong resonance contributions are observed at other scattering angles, with the resonance being approximately $28 \%$ of the interpolated background at $30^{\circ}, 83 \%$ at $90^{\circ}$ and $110 \%$ at $110^{\circ}$. The resonance energy of $4.25 \mathrm{eV}$ and apparent width of $392 \mathrm{meV}$ cited throughout this paper and in Fig. 1, represent the average values determined from Fano profile fits to the data at the two scattering angles, $30^{\circ}$ and $110^{\circ}$, where the resonance was observed and where experimental data were well represented by a Fano shape. At $90^{\circ}$, the uncertainty in the Fano profile fit was higher although the statistical accu- 

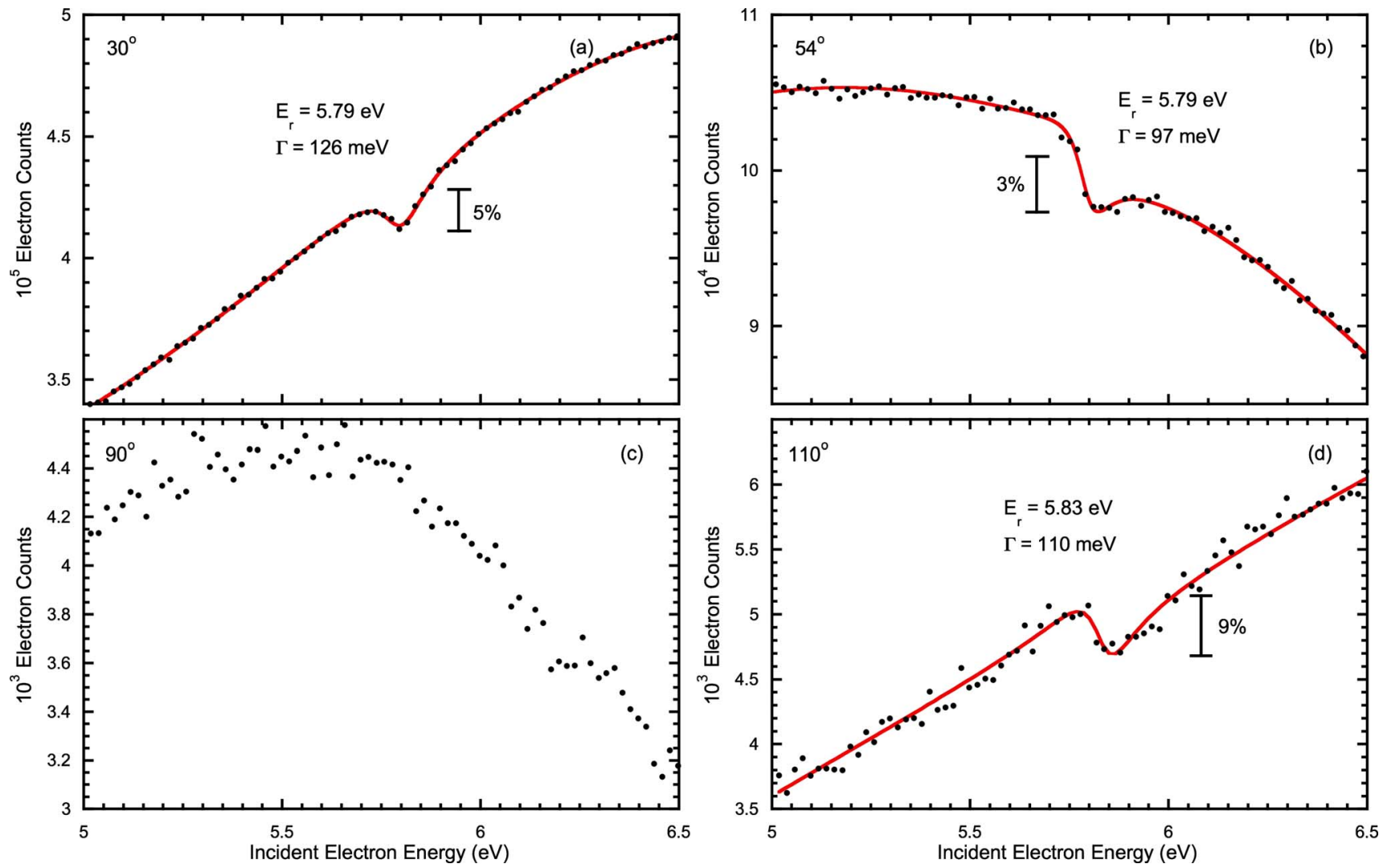

FIG. 3. (Color online) Differential elastic scattering signal in the vicinity of the $5.80 \mathrm{eV}$ cusp or resonance at a scattering angle of (a) $30^{\circ}$, (b) $54^{\circ}$, (c) $90^{\circ}$, and (d) $110^{\circ}$. present data, - a combined polynomial background and Fano profile fit to the resonance structure which obtained the resonance energy and width displayed. The size of the resonance, where present, is also indicated by a vertical bar representing a percentage of the nonresonance background.

racy and relative resonance contributions were still high. Inspection of Fig. 2(c) indicates a departure of the fitted curve from the experimental data points in the low-energy wing of the resonance. This can be an indication that another weak process, just detectable at $90^{\circ}$, affects elastic scattering below $4 \mathrm{eV}$ and causes the overall shape to be different from the expected resonance profile.

Figure 3 shows details of the angular behavior of the structure observed at $5.80 \mathrm{eV}$. The feature is not visible at $90^{\circ}$, which indicates that it has a $p$-wave nature. At other scattering angles, the feature comprises approximately $5 \%$ of the interpolated background at $30^{\circ}, 3 \%$ at $54^{\circ}$ and $9 \%$ at $110^{\circ}$. The energy of $5.80 \mathrm{eV}$ and apparent width of $112 \mathrm{meV}$ represent average values determined from Fano profile fits to the data at the three scattering angles where the resonance was observed. Compared to $30^{\circ}$ and $54^{\circ}$, at $110^{\circ}$ the uncertainty in the Fano profile fit was higher because of the poorer counting statistics which may explain why the resonance energy was $40 \mathrm{meV}$ higher than at the other angles.

Figure 4 shows the details of overlapping resonance structure observed near $7.6 \mathrm{eV}$ in the differential elastic scattering signal at scattering angles of $30^{\circ}, 54^{\circ}, 90^{\circ}$, and $110^{\circ}$. At their peak, the features comprise approximately $1.6 \%$ at $30^{\circ}$ and $54^{\circ}, 15 \%$ at $90^{\circ}$ and $10 \%$ at $110^{\circ}$. The presence of several overlapping resonances prevented Fano profile fitting and, when combined with the poorer statistics compared to the rest of the present data, made it difficult to extract consistent resonance energies and to identify the partial wave behavior of the structures. Instead, to aid interpretation, the data in Fig. 4 are presented with bars marking the energy of structures observed previously in photon excitation [6] and transmission studies [4].

To assist further a discussion of resonances in the energy region near $7.5 \mathrm{eV}$, manipulated transmission data in this energy region are presented in Fig. 5. The energy of the structures observed previously in photon excitation [6] and transmission studies [4] are marked, as are thresholds for the $4 s n l n=4,5,6, l=s, p, d$ configuration states since the transmitted flux is affected by the opening of new channels. As expected, the manipulated transmission data bear the closest resemblance to the differential elastic scattering signal at $30^{\circ}$, the smallest scattering angle observed here. As shown in Figs. 5 and 4(a), both have small dips near $7.5 \mathrm{eV}$ followed by a dominant peak near $7.7 \mathrm{eV}$.

\section{B. Above the ionization threshold}

Above the ionization threshold two resonance regions with distinctly different resonance intensities are observed. The 10-12 eV energy region, where strong resonances were also observed previously in photon excitation functions $[5,7,8]$, was studied in both transmission [electron transmission spectroscopy (ETS)], Fig. 6, and by elastic scattering, Figs. 8(a) and 9. Energies from the present differential elastic 

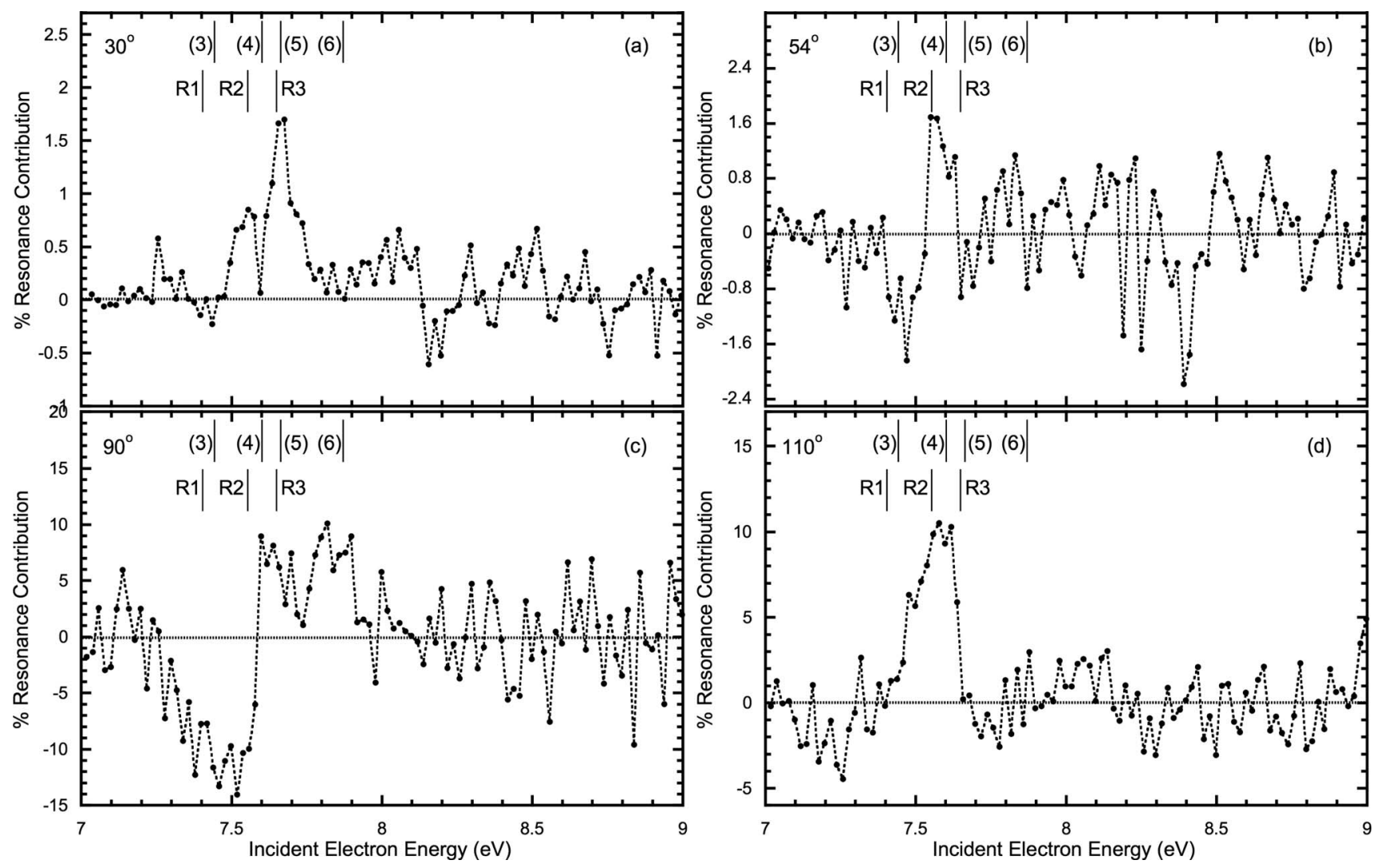

FIG. 4. Percentage resonance contributions to the differential elastic scattering signal in the vicinity of the $7.6 \mathrm{eV}$ resonances at a scattering angle of (a) $30^{\circ}$, (b) $54^{\circ}$, (c) $90^{\circ}$, and (d) $110^{\circ}$. Vertical bars indicate resonance structures observed in previous transmission studies [4] (marked R1, R2, R3) and photon excitation functions [6] (marked with numbers used by authors) which possibly contribute to the structures near $7.6 \mathrm{eV}$ in elastic scattering.

scattering are given in Table II together with energies from the present transmission experiment. Resonances in the 12-17 eV energy region are observed in the transmission spectrum shown in Fig. 7 and corresponding energies are given in Table III.

The derivative of transmitted current between 10 and 12 $\mathrm{eV}$ is presented in Fig. 6, along with the locations of relevant autoionizing states of $\mathrm{Zn}$ with $3 d^{9} 4 s^{2} 4 p$ and $3 d^{10} 4 p^{2}$ configurations $[13,15]$. Above the transmission data are shown scaled Ga energies to be discussed later. The midpoint between the dip and following peak in a derivative structure is normally assigned in ETS to the resonance energy in cases in which the resonance profile corresponds to a pure peak in the total scattering cross section. These are indicated with solid circles. The two lowest energy structures labeled a, $\mathrm{a}^{\prime}$ and $\mathrm{b}$, $b^{\prime}$ are clearly doublets. These are followed by a larger feature consisting of at least three overlapping resonances, $\mathrm{c}, \mathrm{c}^{\prime}$, $\mathrm{c}^{\prime \prime}$, and a smaller resonance, $\mathrm{d}$, near $11.6 \mathrm{eV}$. The large breadth and overlap of $c^{\prime \prime}$ and the uncertainty of its profile makes its ETS energy assignment problematic as discussed in more detail later.

The differential elastic scattering signal measured at $15^{\circ}$ is shown in Fig. 8(a), along with the estimated nonresonance contribution shown as a solid line and the isolated resonance contribution. Four resonance structures are also apparent in the differential elastic scattering signal at $15^{\circ}$. In the three dominant peaks, unresolved doublet and multiplet structure is suggested only by the width of the peaks and the presence of shoulders.

To enable a more direct comparison of the elastic scattering and the transmission data, the latter were manipulated as described above, and the result is presented in Fig. 8(b). The general shape of the manipulated transmission data is in

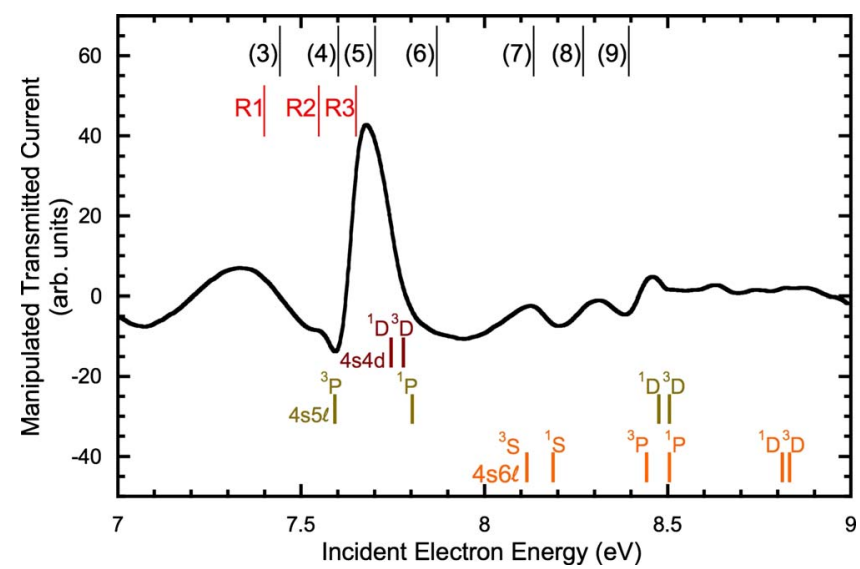

FIG. 5. (Color online) Transmission data between 7 and $9 \mathrm{eV}$ manipulated as described in the text. The thresholds of the $n^{1,3} \mathrm{~S}$, $n^{1,3} \mathrm{P}, n{ }^{1,3} \mathrm{D} n=4,5,6$ states are marked, along with the positions of resonance structures observed by [4] (marked R1, R2, and R3) and [6] (marked with numbers). 
TABLE I. Energies, widths and assignment of resonance structures observed in the angle differential elastic scattering signal below the ionization threshold. The total uncertainty due to energy calibration, energy drifts and the peak fitting is indicated for each structure. Also included are resonance energies determined from photon emission studies [6], electron transmission experiment [4], and BSRM theory [9]. All energies and resonance or apparent width of the structures are in units of eV.

\begin{tabular}{|c|c|c|c|c|c|c|c|c|}
\hline & $30^{\circ}$ & $54^{\circ}$ & $90^{\circ}$ & $110^{\circ}$ & Photons [6] & Transmission [4] & BSRM [9] & Assignment \\
\hline $\mathrm{E}_{r}$ & $4.23 \pm 0.03$ & & $4.29 \pm 0.04$ & $4.26 \pm 0.03$ & & $4.25\left(4 s 4 p^{2}{ }^{2} D\right)$ & $4.234\left(4 s 4 p^{2}{ }^{2} D\right)$ & $4 s 4 p^{2}{ }^{2} D$ \\
\hline$\Gamma$ & $0.405 \pm 0.002^{\mathrm{a}}$ & & $0.46 \pm 0.01^{\mathrm{a}}$ & $0.380 \pm 0.006^{\mathrm{a}}$ & & & 0.372 & \\
\hline $\mathrm{E}_{r}$ & $5.79 \pm 0.03$ & $5.79 \pm 0.03$ & & $5.83 \pm 0.04$ & $5.800 \pm 0.024^{b}$ & $5.79\left(4 s 4 p^{2}{ }^{2} S\right)$ & 5.796 (cusp) & cusp \\
\hline$\Gamma$ & $0.126 \pm 0.008^{a}$ & $0.097 \pm 0.009^{\mathrm{a}}$ & & $0.11 \pm 0.02^{\mathrm{a}}$ & & & & \\
\hline
\end{tabular}

${ }^{\mathrm{a} C o n v o l u t e d}$ with incident beam energy profile.

${ }^{\mathrm{b}}$ From the $4 p{ }^{3} \mathrm{P}_{1}$ photon excitation function [6].

excellent agreement with the differential elastic scattering data at $15^{\circ}$. Doublet structure is still apparent in the manipulated transmission data, however it is not as obvious and highlights the sensitivity of the energy differential experimental technique. Also, the structure near $11.6 \mathrm{eV}$, which is clearly resolved in the differential data, is only a shoulder after integration. Lastly, the width of the structures in the manipulated transmission is much larger than the $30 \mathrm{meV}$ resolution of the experiment, which confirms that the resonances here have a large natural width. Consequently it may be impossible to resolve completely all the resonances with current techniques. Therefore we estimate from a comparison of differential elastic scattering data in Fig. 8(a) and manipulated transmission data in Fig. 8(b) that the a, $\mathrm{a}^{\prime}, \mathrm{b}, \mathrm{b}^{\prime}$ and $\mathrm{c}$, $c^{\prime}$ resonances decay into elastic channel. There is no evidence for a contribution from $\mathrm{c}^{\prime \prime}$ and resonance $\mathrm{d}$ is indicated only at $\theta=15^{\circ}$.

Labels have been placed in Figs. 6-9 to associate resonances in the same group, and also to associate the same resonances seen in different scattering channels, including the photon excitation functions [7]. The same labels are also used in Table II and IV. The resonance energies indicated in Table IV as recommended values have been determined as follows. The energies for resonances labeled $a, a^{\prime}$ and $b, b^{\prime}$, are determined from the derivative of transmitted current. The energies of $c, c^{\prime}$, and $c^{\prime \prime}$ are the average of peak energies observed in photon emission [7], and the energy of the structure labeled $\mathrm{d}$ comes from the peak in the differential elastic scattering signal at $15^{\circ}$.

The differential elastic scattering signal for angles between $30^{\circ}$ and $110^{\circ}$ is shown in Fig. 9. The data are pre- sented in the same manner as the $15^{\circ}$ differential elastic scattering signal with the full line representing the interpolated nonresonance contribution. There is no structure at $54^{\circ}$, which implies a $d$-wave nature for the dominant $\mathrm{b}$ and $\mathrm{c}$ group structures. The structure near $10.8 \mathrm{eV}$ corresponding to $\mathrm{a}, \mathrm{a}^{\prime}$, which cannot be resolved and individually identified, is not clearly visible either at $54^{\circ}$ or at $90^{\circ}$. Absence at $54^{\circ}$ would suggest a $d$-wave while absence at $90^{\circ}$ would suggest a $p$-wave nature for one or both of these two resonances whichever decays into the elastic channel. For the resonance $\mathrm{d}$ it is difficult to distinguish the partial wave behavior because it is relatively weak and masked by contributions from the nearby dominant peak. The peak resonance contributions to the elastic scattering signal are approximately $10 \%$ at $15^{\circ}$ and $30^{\circ}$, and approximately $30 \%$ at $90^{\circ}$ and $110^{\circ}$.

The derivative of the transmitted current in the energy region between 12 and $16.8 \mathrm{eV}$ is shown in Fig. 7. To help in assignment of these features, the positions of selected autoionizing states are marked at energies from references $[13,14]$. The states are identified by their relevant core terms and an excited Rydberg electron. This indicates also the spin orbit splitting in the ion core apparent in the autoionizing states, which is also expected to be reflected in the negative ion spectrum. Energies of observed structures and proposed configurations are given in Table III. The resonance structures in this energy region are weak and their study in the angular differential measurements was not attempted. In this particular case, the advantage of high sensitivity and efficiency of the transmission method, is amply demonstrated. In the scan from 3.5 to $16.8 \mathrm{eV}$, the size of the transmitted current variation due to resonance groups $\mathrm{i}, \mathrm{i}^{\prime}$ and $\mathrm{n}, \mathrm{n}^{\prime}$, was

TABLE II. Energies of resonance structures observed in the differential elastic scattering signal above the ionization threshold. The total uncertainty due to energy calibration, energy drifts and the peak fitting is indicated for each structure observed in elastic scattering. For ETS energies the uncertainty is $\pm 30 \mathrm{meV}$. The letters identifying the resonances are used throughout this paper and in our previous study [7]. All energies are in units of $\mathrm{eV}$.

\begin{tabular}{|c|c|c|c|c|c|c|c|c|c|}
\hline Elastic & $\mathrm{x}$ & $\mathrm{a}$ & $a^{\prime}$ & $\mathrm{b}$ & $\mathrm{b}^{\prime}$ & $\mathrm{c}$ & $\mathrm{c}^{\prime}$ & $\mathrm{c}^{\prime \prime}$ & d \\
\hline $15^{\circ}$ & & $10.75 \pm 0.04$ & & $10.98 \pm 0.04$ & & $11.24 \pm 0.03$ & & & $11.61 \pm 0.04$ \\
\hline $30^{\circ}$ & & $10.75 \pm 0.04$ & & $11.00 \pm 0.04$ & & $11.24 \pm 0.03$ & & & \\
\hline \multicolumn{10}{|l|}{$54^{\circ}$} \\
\hline $90^{\circ}$ & & & & $10.97 \pm 0.04$ & & & $11.33 \pm 0.03$ & & \\
\hline $110^{\circ}$ & & $10.79 \pm 0.04$ & & $11.01 \pm 0.04$ & & & $11.33 \pm 0.03$ & & \\
\hline Transmission & & 10.74 & 10.82 & 11.01 & 11.10 & 11.24 & 11.30 & 11.39 & 11.65 \\
\hline
\end{tabular}




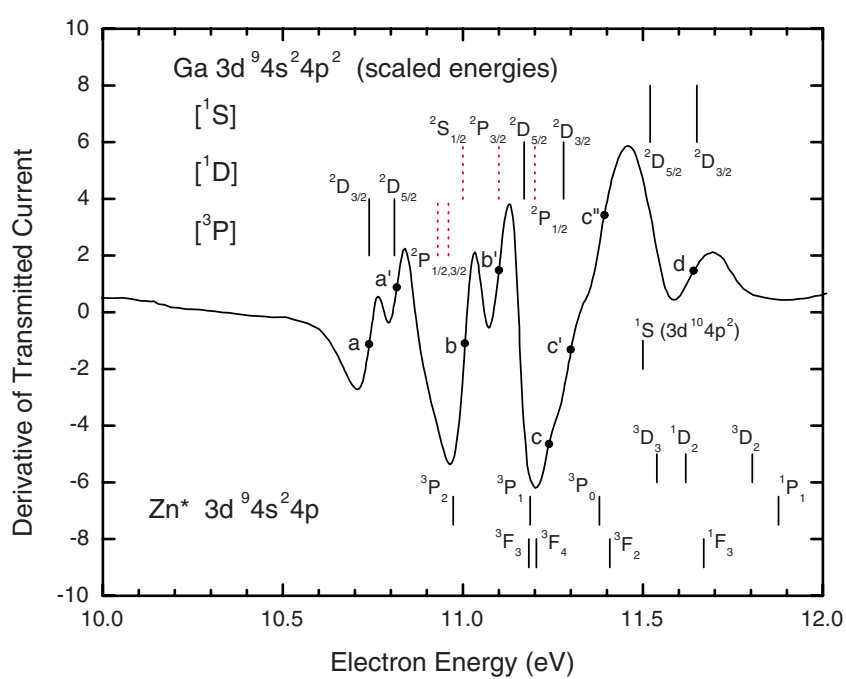

FIG. 6. (Color online) The derivative of the transmitted current for incident electron energies between 10.0 and $12.0 \mathrm{eV}$. The dots indicate resonance energies as determined from the transmission spectrum. For energies indicated by vertical bars see text.

found to be approximately 7 times smaller than the maximum variation in group $\mathrm{c}$ and 22 times smaller than the resonance at $4.25 \mathrm{eV}$. Similarly, the resonance observed above $16 \mathrm{eV}$ in photon excitation function for the $4 s 4 p{ }^{1} \mathrm{P}$ state [7], was very weak, approximately $1 \%$ of the total sig-

TABLE III. Energies (in eV) of resonance structures above 12 $\mathrm{eV}$ observed in electron transmission. Note that the energies refer to the location of extrema in the derivative spectra rather than resonance centers. The proposed electronic configurations are indicated in the table by assigning to each structure (where possible) the parent autoionizing electron configuration and the electron configuration of the outer electrons.

\begin{tabular}{lrcc}
\hline \hline Label & Energy & Parent & Outer electrons \\
\hline $\mathrm{e}$ & 12.30 & & \\
$\mathrm{f}$ & 12.63 & $3 d^{10} 4 p\left({ }^{2} P_{1 / 2}\right) 5 s$ & $5 s^{2}$ \\
$\mathrm{f}^{\prime}$ & 12.79 & $3 d^{10} 4 p\left({ }^{2} P_{3 / 2}\right) 5 s$ & $5 s^{2}$ \\
$\mathrm{~g}$ & 13.15 & $3 d^{10} 4 p 5 p$ & \\
$\mathrm{~h}$ & 13.62 & $3 d^{10} 4 p 4 d$ & $5 s^{2}$ \\
$\mathrm{i}$ & 13.95 & $3 d^{9} 4 s^{2}\left({ }^{2} D_{5 / 2}\right) 5 s$ & $5 s^{2}$ \\
$\mathrm{i}^{\prime}$ & 14.28 & $3 d^{9} 4 s^{2}\left({ }^{2} D_{3 / 2}\right) 5 s$ & \\
$\mathrm{j}$ & 14.47 & $3 d^{10} 4 p\left({ }^{2} P_{1 / 2}\right) 5 d$ & \\
$\mathrm{k}$ & 14.64 & $3 d^{10} 4 p\left({ }^{2} P_{3 / 2}\right) 5 d$ & \\
$\mathrm{l}$ & $\sim$ & \\
$\mathrm{m}$ & 14.71 & & $5 p^{2}$ \\
$\mathrm{n}$ & 14.85 & & $6 s^{2}$ \\
$\mathrm{o}$ & 15.08 & $3 d^{9} 4 s^{2}\left({ }^{2} D_{5 / 2}\right) 5 p$ & $5 p^{2}$ \\
$\mathrm{n}^{\prime}$ & 15.24 & $3 d^{9} 4 s^{2}\left({ }^{2} D_{5 / 2}\right) 6 s$ & $6 s^{2}$ \\
$\mathrm{o}^{\prime}$ & 15.41 & $3 d^{9} 4 s^{2}\left({ }^{2} D_{3 / 2}\right) 5 p$ & \\
$\mathrm{p}$ & 15.56 & $3 d^{9} 4 s^{2}\left({ }^{2} D_{5 / 2}\right) 6 s$ & \\
$\mathrm{q}$ & $\sim 15.84$ & & \\
$\mathrm{r}$ & $\sim 15.98$ & $3 d^{9} 4 s^{2}\left({ }^{2} D_{5 / 2}\right) 6 p$ & \\
\hline \hline & 16.09 & & \\
\hline
\end{tabular}

${ }^{\mathrm{a}}$ This assignment is less certain, see text for discussion.

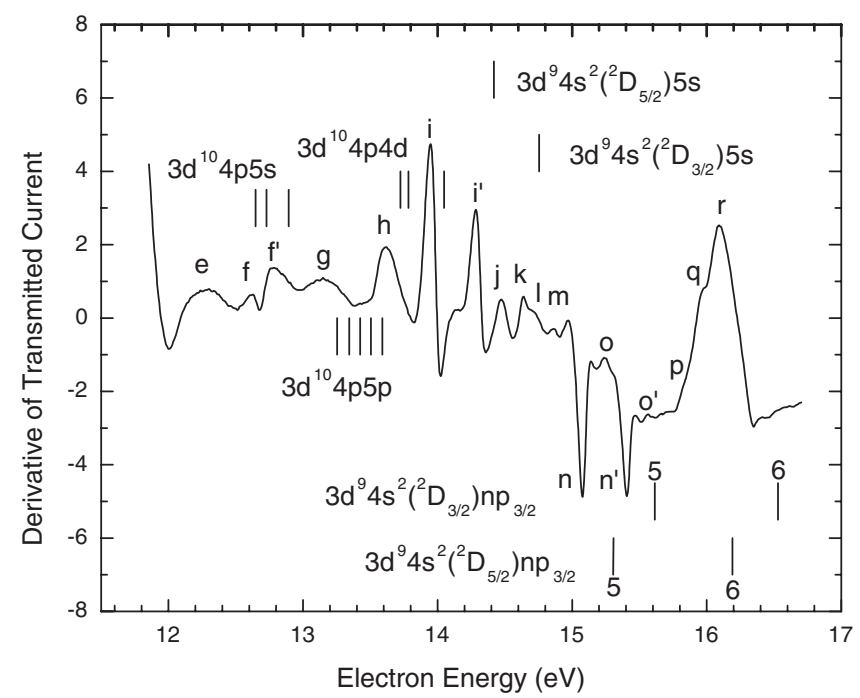

FIG. 7. Derivative of transmitted current between 12 and 16.8 $\mathrm{eV}$, with selected autoionizing state thresholds from $[13,14]$, and resonance structures marked from e to $r$.

nal. The parameters derived from a fit to a Fano profile in this work gave the resonance energy as $16.16 \mathrm{eV}$ and a convoluted width of $460 \mathrm{meV}$. This energy is possibly the best determination of the position of the $\mathrm{r}$ resonance.

\section{DISCUSSION}

\section{A. Below ionization threshold}

Previous experimental $[3,4,6]$ studies in zinc have determined energies and attempted classification of negative ion resonances below the ionization threshold. A parallel study $[3,4]$ of the other group IIB atoms, $\mathrm{Cd}$ and $\mathrm{Hg}$ as well as $\mathrm{Mg}$, an atom with the same $s^{2}$ ground state electronic configuration of the outer orbital but no d orbitals in the core, illustrates the role of the spin orbit interaction with increasing atomic number of the atom. A theoretical BSRM study [9] of the angle-integrated cross section for electron scattering on zinc describes resonance structures up to approximately $8 \mathrm{eV}$ and is generally in good agreement with experiments. However, a few points related to the interpretation of the experimental data and the assignments are still outstanding. These can be resolved in principle by angle differential measurements of intensities for elastic scattering as reported here, and also by inelastic scattering. Apart from one angular study of the resonance at $4.25 \mathrm{eV}$ in elastic scattering in a limited range of scattering angles, $20^{\circ}, 40^{\circ}$, and $55^{\circ}$ [4], such measurements have not been made. The present study extends these measurements and indicates the realistic limits for angular studies imposed by both the apparent density of negative ion states and the nature of the limitations imposed by zinc vapor on the measurements.

The early experimental study of temporary negative ions in $\mathrm{Mg}, \mathrm{Zn}, \mathrm{Cd}$, and $\mathrm{Hg}$ [3] established the existence of a large shape resonance at low impact energy and assigned it as $\mathrm{n} s^{2} \mathrm{n} p{ }^{2} \mathrm{P}$. In zinc, the shape resonance is observed at $0.49 \pm 0.03 \mathrm{eV}$ in relatively good agreement with BSRM 

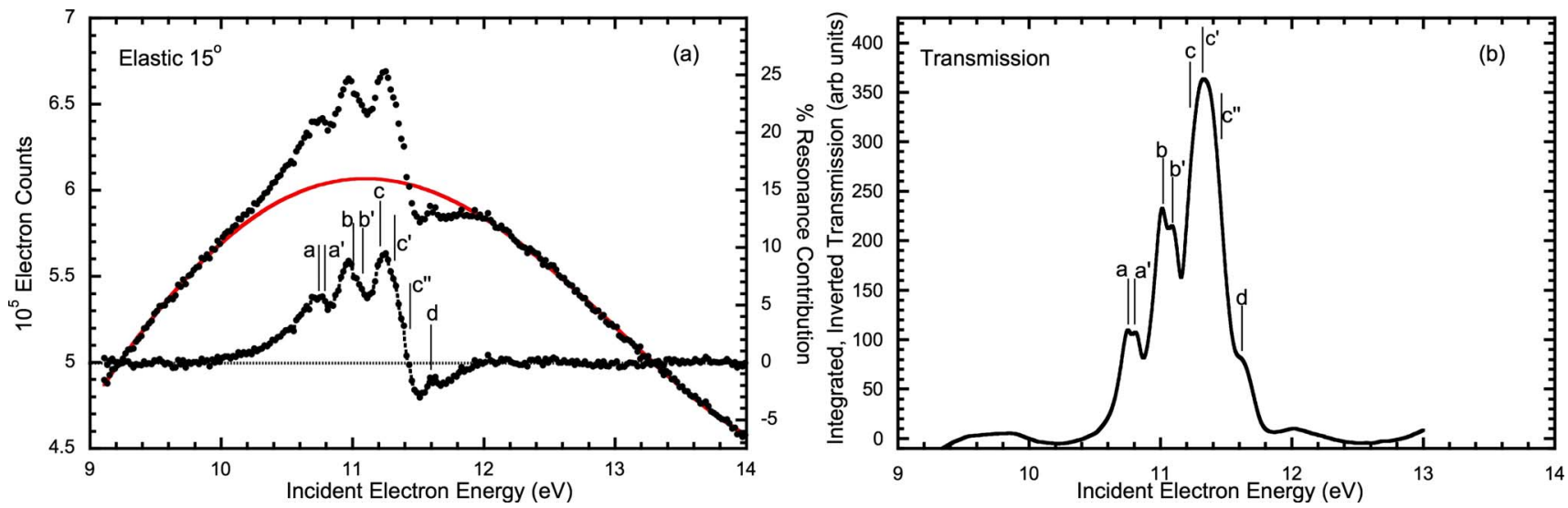

FIG. 8. (Color online) (a) Differential elastic scattering: $\cdots$, signal at $15^{\circ}$, upper curve and the percentage resonance contribution to the data, lower curve; full red line, a polynomial fit to the slowly varying nonresonance background. (b) Transmission data manipulated as described in the text. The letters indicated the average energies of resonance structure peaks observed in both the present experiment and [7].

calculations [9], which placed it at $0.707 \mathrm{eV}$ with a predicted width of $\Gamma=1140 \mathrm{meV}$. The present study was not able to obtain reliable data at these low energies.

The broad resonance at $4.25 \mathrm{eV}$, shown in Fig. 1 and in more detail in Fig. 2, located near the excitation thresholds of the ${ }^{3} \mathrm{P}_{0,1,2}$ states at 4.006, 4.030, and $4.078 \mathrm{eV}$, has been observed previously in electron transmission and differential elastic scattering studies [4] and was indicated also by the BSRM calculations [9]. As seen in Fig. 1, this is the most pronounced resonance structure below the ionization threshold. The energy of the resonance in the present experiment is
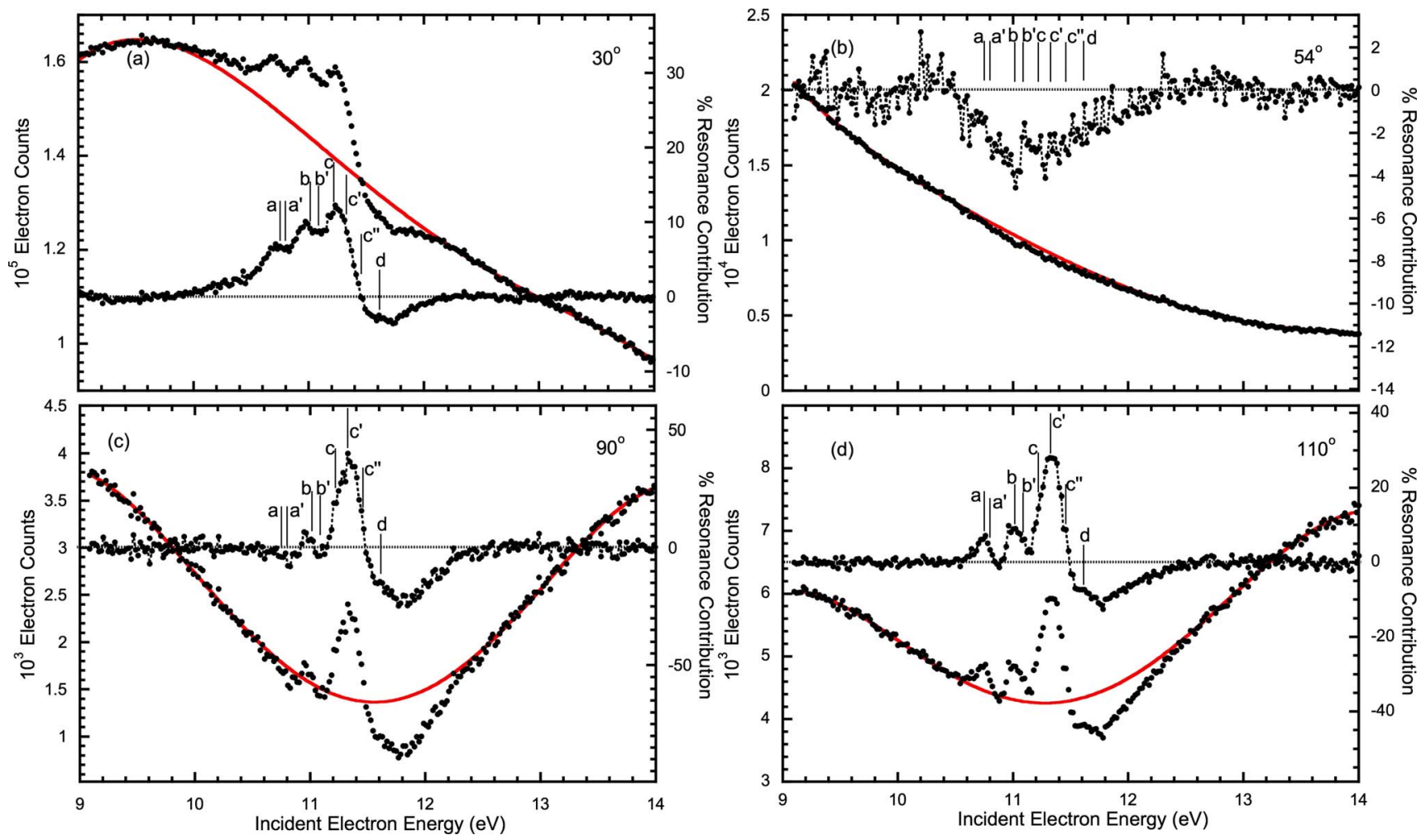

FIG. 9. (Color online) Differential elastic scattering at (a) $30^{\circ}$, (b) $54^{\circ}$, (c) $90^{\circ}$, and (d) $110^{\circ}$. $\cdots$, Measured intensities and the percentage resonance contribution to the data. Red full line represents a polynomial fit to the slowly varying nonresonance background, subtracted from measured intensities to obtain resonance contributions. The letters indicate the recommended energies from Table IV. in good agreement with these previous studies as seen in Table I. The shape of the resonance structure observed here is a convolution of a Fano profile and Gaussian apparatus function. As a consequence the experimental width of the structure is larger than the natural width $\Gamma=372 \mathrm{meV}$ indicated by BSRM theory. The departure of the fitted curve from the experimental data in the low energy wing of this resonance in the spectrum at $90^{\circ}$ is consistent with the weak resonance process at $3.82 \mathrm{eV}$ observed in transmission [4].

As in the previous differential elastic scattering experiment, the present measurements do not show a resonance 
TABLE IV. Energies of $3 d^{9} 4 s^{2} 4 p^{2}$ autoionizing states of isoelectronic atom Ga and these states scaled as described in text, compared with recommended energies of corresponding negative ion states in zinc. Tentative assignments shown are based on this comparison and observed angular behavior of resonance decay into elastic channel.

\begin{tabular}{|c|c|c|c|c|c|c|c|c|c|c|c|}
\hline $\mathrm{Ga}$ & ${ }^{3} P:{ }^{2} D_{3 / 2}$ & ${ }^{2} D_{5 / 2}$ & ${ }^{2} P_{1 / 2}$ & ${ }^{2} P_{3 / 2}$ & ${ }^{1} D:{ }^{2} S_{1 / 2}$ & ${ }^{2} P_{3 / 2}$ & ${ }^{2} P_{1 / 2}$ & ${ }^{2} D_{5 / 2}$ & ${ }^{2} D_{3 / 2}$ & ${ }^{1} S:{ }^{2} D_{5 / 2}$ & ${ }^{2} D_{3 / 2}$ \\
\hline E & 18.898 & 19.131 & 19.506 & 19.588 & 19.727 & 20.033 & 20.325 & 20.227 & 20.588 & 21.299 & 21.698 \\
\hline $\mathrm{E}_{\text {scaled }}{ }^{\mathrm{a}}$ & 10.74 & 10.81 & 10.93 & 10.95 & 11.00 & 11.09 & 11.18 & 11.15 & 11.27 & 11.49 & 11.61 \\
\hline \multicolumn{12}{|l|}{$\mathrm{Zn}^{-}$} \\
\hline $\mathrm{E}_{\text {recom. }}{ }^{\mathrm{b}}$ & $10.74^{c}$ & $10.82^{\mathrm{c}}$ & & & $11.01^{\mathrm{c}}$ & $11.10^{c}$ & $11.22^{\mathrm{d}}$ & & $11.33^{\mathrm{d}}$ & $11.46^{\mathrm{d}}$ & $11.61^{\mathrm{e}}$ \\
\hline Label & $\mathrm{a}$ & $\mathrm{a}^{\prime}$ & & & b & $\mathrm{b}^{\prime}$ & $\mathrm{c}$ & & $c^{\prime}$ & $c^{\prime \prime}$ & $\mathrm{d}$ \\
\hline Assign. ${ }^{\mathrm{f}}$ & ${ }^{3} P:{ }^{2} D_{5 / 2}$ & ${ }^{2} D_{3 / 2}$ & & & ${ }^{1} D:{ }^{2} D_{5 / 2}$ & ${ }^{2} D_{3 / 2}$ & ${ }^{1} S:{ }^{2} D_{5 / 2}$ & & ${ }^{2} D_{3 / 2}$ & $3 d^{9} 4 s^{2} 4 p 4 d$ & $3 d^{9} 4 s^{2} 4 p 4 d$ \\
\hline
\end{tabular}

${ }^{\mathrm{a}} \mathrm{Ga} 3 d^{9} 4 s^{2} 4 p^{2}$ autoionizing state energies scaled to the interval spanned by the recommended $\mathrm{Zn}^{-}$values.

${ }^{\mathrm{b}} \mathrm{Zn}^{-}$energies, recommended values.

${ }^{\mathrm{c}}$ From transmission, present work.

${ }^{\mathrm{d}}$ Mean values from photon emission [7].

${ }^{\mathrm{e}}$ Present elastic scattering at $\theta=15^{\circ}$.

${ }^{\mathrm{f}} \mathrm{See}$ text for discussion.

feature at $54^{\circ}$, which implies that the negative ion decays to the ground state by the emission of a $d$-wave electron. This restricts the possible negative ion states observed in the elastic scattering channel to only those with positive parity and total angular momentum of either $J=5 / 2$ or $J=3 / 2$. Conservation of parity suggests a $3 d^{10} 4 s 4 p^{2}$ configuration, which through $L S$-coupling leads to four states, which obey the conservation of total angular momentum $J$, namely, $4 s\left({ }^{2} S\right) 4 p^{2}\left({ }^{3} P\right)^{4} P_{5 / 2,3 / 2}$ and $4 s\left({ }^{2} S\right) 4 p^{2}\left({ }^{1} D\right)^{2} D_{5 / 2,3 / 2}$. Of these states, the ${ }^{2} D_{5 / 2,3 / 2}$ is more likely since it complies with the pure $L S$-coupling requirement that $\Delta L=\Delta S=0$ [16]. This leads to assignment of the strong resonance at $4.25 \mathrm{eV}$ as $4 s 4 p^{2}{ }^{2} \mathrm{D}$. This is in agreement with the assignment from previous experimental and theoretical studies.

The origin of the weak structure at $3.82 \mathrm{eV}$ is highlighted by the comparison of data for different atoms. No corresponding structure is observed in magnesium [4], a light atom well described within the $L S$ coupling approximation. However the strength and complexity of the shape and the number of observed structures progressively increases from zinc to mercury, in accord with the increase of the spin-orbit interaction [4]. In zinc, the structure is very weak, in cadmium two clear structures are detected, while in mercury, where fine structure caused by spin-orbit interaction is easy to resolve, a well-developed resonance structure is associated with individual fine structure components of the $6 s 6 p{ }^{3} \mathrm{P}_{0,1,2}$ states [17]. Following this comparison the lower weak resonances in zinc and cadmium, where the predominant coupling is $L S$, have been assigned as $n s n p^{2}{ }^{4} \mathrm{P}_{3 / 2,5 / 2}$ negative ions where $n=4$ for zinc and $n=5$ for cadmium [4]. The appearance of these resonances still complies predominantly with $L S$ coupling.

Figure 3 shows the angular behavior of a sharp feature which coincides almost exactly with the $4 s 4 p{ }^{1} P$ threshold at $5.796 \mathrm{eV}$. The structure was also observed in previous transmission studies [4], as well as in the photon excitation function of the $4 s 4 p{ }^{3} \mathrm{P}_{1}$ state [6]. Similar structures have also been observed for cadmium at the $5.417 \mathrm{eV}$ threshold of the $5 s 5 p{ }^{1} P$ state, and for mercury at the $6.702 \mathrm{eV}$ threshold of the $6 s 6 p{ }^{1} P$ state $[1,4,18]$. The assignment of the structure is not clear. Early transmission studies concluded on the basis of shape that the structure is a cusp associated with the opening of the $4 s 4 p{ }^{1} \mathrm{P}$ excitation channel. This assignment has been strengthened recently in a time delay analysis with BSRM calculations [9].

The comparative study of resonances in $\mathrm{Mg}, \mathrm{Zn}, \mathrm{Cd}$, and $\mathrm{Hg}$ using different experimental methods has led to the conclusion that this structure is most likely a $n s n p^{2}{ }^{2} S$ negative ion resonance where $n=4$ for zinc, $n=5$ for cadmium and $n=6$ for mercury [4]. This conclusion seems to be supported, for example, by the shape observed in the photon excitation function of $5 s 5 p{ }^{1} \mathrm{P}$ state in $\mathrm{Cd}$ which is consistent with a threshold resonance. Also the same authors conclude from observation of a weak resonance structure in differential elastic scattering at $90^{\circ}$, that the resonance decay is consistent with an $s$-wave. In zinc a structure is also predicted [9], although not associated with a resonance, at the threshold for excitation of the $4 s 4 p{ }^{1} \mathrm{P}$ state, while a lower resolution photon excitation function of this state is not conclusive, i.e., the cross section shape is consistent with a threshold enhancement but much less pronounced than predicted by theory. In $\mathrm{Mg}$, the structure appears slightly below the corresponding ${ }^{1} \mathrm{P}$ threshold [4].

The cusp assignment in zinc is strengthened also by the present differential measurements for elastic scattering in contrast with observations in $\mathrm{Cd}$. We observe the absence of the feature at $90^{\circ}$, which indicates that it is associated with $p$-wave electrons and cannot be due to a $4 s 4 p^{2}{ }^{2} S$ resonance as suggested [4]. It is this partial wave which is responsible for near-threshold excitation of the $4 s 4 p{ }^{1} P_{1}$ state. Note, however, that within the statistical accuracy of the data a Fano shape characteristic for a negative ion resonance is fitted very well to the present experimental data as can be seen in Fig. 3. One possibility is that there is a resonance at the threshold for excitation of ${ }^{1} \mathrm{P}$ states in all the IIB group atoms, while a cusp is observed in elastic scattering on the opening of this scattering channel and possibly in excitation of the $n{ }^{3} \mathrm{P}_{0,1,2}$ states.

The complex structure in elastic scattering between 7 and $8.5 \mathrm{eV}$, shown in Fig. 4, appears to be influenced by a num- 
ber of different resonances. BSRM calculations [9] predict 11 resonances in this energy region, built on the parent configurations of $4 s 5 p, 4 s 4 d, 4 s 6 s, 4 s 7 s$, and $4 s 5 d$, and having a range of different symmetries. Multiple resonances in this energy region have also been identified in electron transmission studies [4], and our previous photon excitation function [6], which indicates selective angular momentum dependent decay of the resonances in this energy region.

The comparison of elastically scattered electron intensities in Fig. 4(a) and in the manipulated transmission data in Fig. 5 indicates a significant similarity in general shape in the 7-9 eV energy region although the statistical accuracy of data in Figs. 4(a) is not very good. The strongest resonances in transmission [4], marked R1, R2, and R3, and in optical emission [6] marked (3), (4), and (5) in Figs. 4(a)-4(c), are also observed in angular differential elastic scattering. Apparent differences in resonance energies from transmission and photon emission experiments of $40 \mathrm{meV}$ (3-R1), $50 \mathrm{meV}$ (4-R2) and $10 \mathrm{meV}$ (5-R3), are comparable to uncertainties in their determination in different experiments and might not indicate that they are different structures as considered previously [6]. It is difficult to determine energies more precisely when resonances overlap or are closely spaced and do not appear as clear Fano profiles. Note however that all data below the ionization threshold are scanned in a continuous spectrum and that the same calibration uncertainty observed for lower resonances applies for higher resonances.

Sullivan et al. [4] have based their assignment of higher resonances in the group IIB atoms $\mathrm{Zn}$ and $\mathrm{Cd}$ and also in $\mathrm{Mg}$, on observed regularities and repetition of resonance structure with increasing energy, i.e., increasing the principal $n$ quantum number of the electrons in the negative ion. They used a detailed knowledge of lower members to assign the configuration and symmetry of higher resonances. However, these assignments are not in agreement with the prediction of BSRM calculations [9]. Further comment is limited since neither data from different experiments nor BSRM theory have an accuracy suitable to examine the complexity of these higher structures.

Further discussion is warranted for the lowest resonance in Figs. 4(a)-4(c), which is observed as a broad structure in transmission, marked as R1 and assigned by Sullivan et al. [4] as $4 s 5 s 4 d$, and in optical emission studies marked (3). This resonance appears in elastic scattering as a broad dip particularly prominent at a scattering angle of $90^{\circ}$, Fig. 4(c). It was observed at an energy of $7.44 \mathrm{eV}$ in photon emission from the $4 p{ }^{1} \mathrm{P}_{1}$ state but not ${ }^{3} \mathrm{P}_{0,1,2}$ [6]. The overall cross section shape measured for emission of photons from the decay of the $4 p{ }^{1} \mathrm{P}_{1}$ state [6] was in very good agreement with the prediction from the BSRM theory [9] in an energy range including the resonances (3), (4), and (5) (see Fig. 6(b) of reference [6]). Also, the BSRM calculations have reproduced the fine details of the electron transmission spectrum. However, no resonances were identified by BSRM at nearby energies or with a width appropriate for the structure at 7.44 $\mathrm{eV}$. A narrow $4 s 4 d 5 s^{2} \mathrm{D}$ resonance is predicted at $7.725 \mathrm{eV}$. This situation is indicative of limitations which can be expected when comparing experiment and theory at energies above $7 \mathrm{eV}$. In elastic scattering a resonance with a $4 s 5 s 4 d$ configuration should appear in a $d$-wave and disappear at $54^{\circ}$. We still observe a dip at $\theta=54^{\circ}$ and the angular behavior is more in line with a $s$-wave behavior and does not support the tentative assignment of Sullivan et al. [4].

Although the present angular differential elastic scattering data in Fig. 4 do not provide a basis for a definitive assignment of resonances (4) and (5) (R2 and R3), some insight can be obtained from application of parity conservation to elastic data and comparison with theoretical predictions. Modeling of differential cross section for elastic scattering in this energy range would be very useful in the final assignment of these resonances.

\section{B. Above ionization threshold}

\section{10-12 eV energy region}

The present study of negative ion resonances in the lowest autoionizing region of zinc has provided the most complete information on the number of negative ion states and their energies by using different techniques and encompassing different decay channels. This enables a set of recommended values based on present and previous work $[2,5,7]$ to be established as given in Table IV. However we note that due to the breadth and partial overlapping of resonance structure, the true number of resonance states, in particular the existence of even broader ones, cannot be ruled out. As discussed in the next subsection, the benefits of high sensitivity and good energy resolution of the transmission measurements have enabled the most complete mapping of the energy resonances above $12 \mathrm{eV}$, which is important for spectroscopic assignments and for the exploration of possible changes in coupling schemes that may occur between the $n=4$ and $n=5$ resonance states.

Also reported here is the first study of the angular behavior of the resonance structures in the 10-12 eV energy range as given in plots (a) to (d) in Fig. 9 and also Fig. 8(a). Angular behavior in purely elastic scattering can assist in identification of the parity and angular momentum of a resonance if the partial wave is the same for the incident and the outgoing electrons which, in some favorable cases, can be experimentally observed. As can be seen in the spectrum of Fig. 9(b) the resonance structure almost completely disappears at $54^{\circ}$, indicating that all the resonances observed to decay into the elastic channel do so by the emission of a $d$-wave electron. This implies that these resonances have even parity, and a total-angular momentum of $J=3 / 2$ or $5 / 2$. The configurations with even parity that lie in the vicinity of the resonances include $3 d^{9} 4 s^{2} 4 p^{2}$, and at somewhat higher energies $3 d^{10} 4 p^{2} 4 d$ and $3 d^{9} 4 s^{2} 4 p 4 f$. Judging from the energy of the corresponding autoionizing states as tabulated in the NIST database [14], i.e., $3 d^{10} 4 p\left({ }^{2} P_{3 / 2}\right) 4 d$ at $14.031 \mathrm{eV}$ and the $3 d^{9}\left({ }^{2} D_{5 / 2}\right) 4 s^{2} 4 f$ at $16.309 \mathrm{eV}$, the latter two are expected at higher energy. One resonance of Fig. 6, feature $c^{\prime \prime}$, does not seem to appear in the elastic decay channel, and thus it cannot be concluded that its parity must be even.

Resonance assignments and the appropriate momentum coupling scheme for the $10-12 \mathrm{eV}$ resonance region were previously discussed by Napier et al. [7]. That discussion introduced a comparison with the corresponding autoionizing states of the isoelectronic gallium atom in its $3 d^{9} 4 s^{2} 4 p^{2}$ 
configuration. Here, we consider this approach in more detail. To make a closer comparison, we scaled the energy span of the relevant $\mathrm{Ga}$ states to the one encompassing the a-d negative-ion resonances in zinc and shifted them so that the lowest states agree in energy. This scheme means, of course, that the agreement in energy of the lowest and highest of the resonances, that is, features a and d, is not significant. For this we used the gallium excited state energies from the NIST database [14]. Unfortunately, only the optically allowed transitions are provided. The Ga and scaled Ga energies and the corresponding state symmetries are given in Table IV and the scaled energies shown in Fig. 6. We note that the scaled $\mathrm{Ga}$ energies can only be considered a rough approximation as there is uncertainty in the assignment of the upper member of the manifold of resonance states. More significantly, the nature of the outer $4 p^{2}$ orbitals is intrinsically more diffuse in $\mathrm{Zn}^{-}$than in $\mathrm{Ga}$ and this will affect the interaction energies.

The gallium model and determination of the most appropriate coupling scheme is based on the assumption that electrostatic interaction between the two $4 p$ electrons is larger than the noncentral part of the interaction with the $3 d^{-1}$ ion core, which is again larger than the spin orbit interaction in the ion core and between the pair. The first assumption is reasonable as the $4 p$ pair is far enough from the ion core and screened by the isotropic charge distribution in the filled $4 s$ subshell. This leads then to LS coupling for the outer two electrons to obtain the ${ }^{3} P,{ }^{1} D$, and ${ }^{1} S$ states with subsequent LS coupling to the ${ }^{2} D_{5 / 2,3 / 2}$ ion core [19] as indicated in Fig. 6. The comparison given in Fig. 6 between the ETS data and the scaled Ga energies shows, at first glance, a striking match between certain of the features. Nevertheless, after a more complete analysis outlined below, we have concluded that this agreement appears to be fortuitous. We indicate with solid vertical lines the scaled Ga energies and states that we associate with the resonances and use dashed lines for the remainder.

The splitting in the lowest resonance pair, a, a' corresponds well to the spacing of the two fine structure components of the lowest $\left[{ }^{3} P\right]^{2} D_{3 / 2,5 / 2}$ scaled autoionizing states of gallium derived from the ${ }^{3} P$ state of the $4 p^{2}$ electron pair. Both of these fine structure components have the correct parity and can decay into the elastic channel by emission of a $d$-wave electron. Although it is tempting to assign the resonance a to $j=3 / 2$ and $a^{\prime}$ to $j=5 / 2$, consistent with the scaled $\mathrm{Ga}$ values, the magnitude of a appears larger than that of $\mathrm{a}^{\prime}$, particularly in the integrated transmission data. This suggests the possibility that the assignment is reversed from that in $\mathrm{Ga}$ and that the $\mathrm{j}=5 / 2$ state lies lower, as observed in the ${ }^{2} D$ states arising from the ${ }^{1} D$ and ${ }^{1} S$ couplings of the $4 p^{2}$ electrons. It should be noted, however, that the apparent sizes of the resonances could also be affected by differences in lifetime if their natural widths are less than the electron beam resolution, which is not the case here. Tentatively, we propose that the $j=5 / 2$ component lies lower.

Although the energies of resonances $b$ and $b^{\prime}$ coincide well with two of the scaled Ga energies, namely those of the $\left[{ }^{1} D\right]^{2} S_{1 / 2}$ and ${ }^{2} P_{3 / 2}$ states, this assignment conflicts in part with the conclusions from the differential elastic scattering results in which we exclude the $j=1 / 2$ states. Thus the agreement with these Ga states in Table IV is accidental, and we must examine other fine structure pairs. Because of the similarity in resonance profiles of $\mathrm{a}, \mathrm{a}^{\prime}$ and $\mathrm{b}, \mathrm{b}^{\prime}$, the best candidates for the latter seem to be the $\left[{ }^{1} D\right]^{2} D_{5 / 2,3 / 2}$ states. These scaled Ga states lie 140 and $170 \mathrm{meV}$ above b and b', respectively. In both the ET spectrum of Fig. 6 and the integrated transmission spectrum of Fig. 8(b), resonance b is clearly larger than $b^{\prime}$, consistent with the overall $j$ value of $5 / 2$ compared to $3 / 2$.

Assignment of the $\mathrm{c}, \mathrm{c}^{\prime}, \mathrm{c}^{\prime \prime}$, and $\mathrm{d}$ resonances presents a more difficult problem, stemming from the close spacing of $c$ and $\mathrm{c}^{\prime}$ and the breadth of $\mathrm{c}^{\prime \prime}$. We note that $\mathrm{c}$ and $\mathrm{c}^{\prime}$ generally seem to behave similarly, are of comparable size, and track together in the differential measurements. We note also that features $c^{\prime \prime}$, particularly, and d, to some extent, are broader than the other resonances in the electron transmission spectrum of Fig. 6. The larger breadth argues for the possibility that these resonances decay strongly into the autoionizing states of the $\mathrm{Zn}^{*} 3 d^{9} 4 s^{2} 4 p$ configuration. Strong support for this appears in measurements of the electron impact ionization efficiency of Zn by Hashizume and Wasada [20], which display a strong peak at $11.45 \mathrm{eV}$ that we associate with $\mathrm{c}^{\prime \prime}$ and a smaller peak near $11.67 \mathrm{eV}$ visible in their data but not tabulated, which we attribute to resonance d. Similar results, but with lower resolution were also obtained by Shpenik et al. [21]. We note here that the discrepancy between the ETS energy for $\mathrm{c}^{\prime \prime}$ and that determined from photon emission studies [7] could arise from the uncertainty in determining the resonance profile in the ETS data.

While we favor assignment of two of the resonances $\mathrm{c}, \mathrm{c}^{\prime}$, $\mathrm{c}^{\prime \prime}$, and do the $\left[{ }^{1} S\right]^{2} D_{5 / 2,3 / 2}$ states, the choice is not clear. We offer possible conjectures that will have to be further explored either by additional experimental data or scattering calculations. Based on our assignment of $b, b^{\prime}$, the $\left[{ }^{1} S\right]^{2} D_{5 / 2,3 / 2}$ states in the scaled Ga spectrum may also be shifted to lower energy in $\mathrm{Zn}^{-}$. To line up with $\mathrm{c}$ and $\mathrm{c}^{\prime}$, the shift would be roughly twice the size of that invoked for $b$, $\mathrm{b}^{\prime}$. This leaves the $\mathrm{c}^{\prime \prime}$ and $\mathrm{d}$ features to be assigned. Because of the different decay characteristics of $c^{\prime \prime}$ and its apparent absence in the elastic channel, there is ample reason to assume that it does not belong to the $3 d^{9} 4 s^{2} 4 p^{2}$ configuration. Its strong presence in the ionization measurements [19] suggests that it can decay efficiently into the autoionizing states shown in Fig. 6 of the $3 d^{9} 4 s^{2} 4 p$ configuration. Thus we propose that states derived from the odd parity $\mathrm{Zn} 3 d^{9} 4 s^{2} 4 p 4 d$ configuration may account for $\mathrm{c}^{\prime \prime}$ and possibly $\mathrm{d}$.

An additional feature, not discussed so far, is the broad undulation of the cross section in the energy region from approximately 10 to $13 \mathrm{eV}$, the shape of which varies with scattering angle as indicated by the smooth fitted line in spectra (a)-(d) in Fig. 9. Resonances a-c are superimposed on this broad structure. Interestingly, in scattering at $54^{\circ}$, where the resonances disappear, the broad structure, although weak, is still visible in the shape of a peak followed by a dip. Although no attempt was made to correct the detected signal for variations caused by electron optics, we note that observations in different excitation channels and in different experiments $[7,8]$ are consistent with the existence of this broad variation. This suggests the possible existence of a shape resonance. 


\section{12-17 eV energy region}

Figure 7 displays the electron transmission spectrum at the highest energies reported here. Because of the difficulty of locating the resonance energies of each feature without knowledge of the profiles, we label each feature with a letter placed at the positive extremum in the derivative signal (the negative extremum in the case of $\left.n, n^{\prime}\right)$. For reference, the energies of these extrema are reported in Table III along with the configurations of the excited neutral "parent" states of $\mathrm{Zn}$, shown by vertical lines in Fig. 7, and possible assignments of the configurations of the outer two electrons of the temporary negative ion state.

The lowest features, $\mathrm{f}, \mathrm{f}^{\prime}, \mathrm{g}$, and $\mathrm{h}$, appear to be associated with the three doubly excited state configurations having the filled $3 d$ shell. In particular, we note that features $f$ and $\mathrm{f}^{\prime}$ are separated by $0.16 \mathrm{eV}$, which is close to the $3 d^{10} 4 p\left({ }^{2} P_{1 / 2,3 / 2}\right) 5 s$ splitting of $0.13 \mathrm{eV}$ of the parent neutral states. An outer configuration of $5 s^{2}$ for the resonances seems likely. The feature labeled e may not be a resonance, since it could appear as a consequence of the dip on the high-energy side of the d resonance located below $12 \mathrm{eV}$. The smaller features labeled $\mathrm{j}$ and $\mathrm{k}$ lie close to possible parent states such as $3 d^{10} 4 p\left({ }^{2} P_{1 / 2,3 / 2}\right) 5 d$ at 14.522 and $14.684 \mathrm{eV}$, which are not shown in Fig. 7 to avoid crowding. Similarly, features 1 and $m$ may derive from $3 d^{10} 4 p\left({ }^{2} P_{1 / 2,3 / 2}\right) 6 d$.

The most striking features in Fig. 7 are the narrow resonance doublets labeled $\mathrm{i}, \mathrm{i}^{\prime}$ and $\mathrm{n}, \mathrm{n}^{\prime}$. The splitting in each pair is $0.33 \mathrm{eV}$, which corresponds very well to the $0.337 \mathrm{eV}$ spacing in the ${ }^{2} D_{5 / 2^{-}}{ }^{2} D_{3 / 2}$ ion core to which the pair of electrons can be considered to be bound. The resonance profiles of $i$ and $i^{\prime}$ consist of a shallow dip and large peak followed by a deeper dip on the high-energy side. This suggests that the resonance energy is perhaps $30 \mathrm{meV}$ above the labeled peaks. Such a profile has both a constructive and destructive contribution in the total scattering cross section and must therefore correspond to electron scattering in a relatively low-partial wave in which interference with the nonresonant background in this partial wave is possible. The derivative profiles of $n$ and $n^{\prime}$, on the other hand, are almost inverted relative to those of $i$ and $i^{\prime}$. This implies that the partial wave cannot be the same as the lower lying doublet. The binding energy of the $\mathrm{i}, \mathrm{i}^{\prime}$ pair to the $\mathrm{Zn}$ excited $3 d^{9}\left({ }^{2} D_{5 / 2,3 / 2}\right) 4 s^{2} 5 s$ states, $0.43 \mathrm{meV}$, is typical of that associated with Rydberg resonances having $n s^{2}$ outer electrons, and thus we identify them with the $3 d^{9} 4 s^{2} 5 s^{2}$ electron configuration. Similar considerations for the $\mathrm{n}, \mathrm{n}^{\prime}$ pair argue for the resonance energies to lie slightly below the negative going peaks in the derivative. The resonances are bound by slightly less, $0.25 \mathrm{eV}$, to the $3 d^{9} 4 s^{2}\left({ }^{2} D_{3 / 2,5 / 2}\right) 5 p$ excited states and we associate them with the $3 d^{9} 4 s^{2} 5 p^{2}$ configuration. Finally, the very small features o and $\mathrm{o}^{\prime}$ appear to have the same splitting and may also derive from the same configuration.

The autoionizing states of $\mathrm{Zn}$ are well known to undergo a change in coupling scheme between the $3 d^{9} 4 s^{2} 4 p$ configuration and higher $3 d^{9} 4 s^{2} n p$ configurations [13,22]. It was shown that the precise interpretation of the optical spectrum, most recently using synchrotron radiation, must be based on the LS coupling for the $3 d^{9} 4 s^{2} 4 p$ states, but $j, K$ for the
$3 d^{9} 4 s^{2} 5 p$ (see Sommer et al. [22] and references therein). Consequently, it is reasonable to expect that temporary negative ion states based on configurations containing more Rydberg-like orbitals exhibit similar effects. This alternative coupling would be $(\mathrm{j}, \mathrm{L})$ where the two outer electrons couple to form a resulting orbital momentum $\vec{L}$ and spin $\vec{S}$. In the next step, $\vec{L}$ couples with the $\vec{j}$ of the ion core to form $\vec{K}$, which then couples to the spin $\vec{S}$ of the pair to obtain a resultant $\vec{J}$. For this coupling to occur, the electrostatic interaction must be weak compared with spin orbit interaction of the ion core, but strong compared with spin-orbit coupling of external electrons. A further consideration in applicability of the $(\mathrm{j}, \mathrm{L})$ coupling are exchange forces, which act on electron spin as discussed by Racah [23]. A pair of energy levels is usually observed and an application of this coupling was already used in discussion of negative-ion states of noble gases (see for example [24,25]).

The remaining very pronounced feature in Fig. 7, labeled $\mathrm{p}, \mathrm{q}$, and $\mathrm{r}$, consists of a broad composite structure situated below the $6 p$ states near $16 \mathrm{eV}$. This feature has a certain superficial resemblance to that of $c, c^{\prime}$, and $c^{\prime \prime}$ in Fig. 6, however, as in that case also, the assignment is uncertain. Similarly to the $n=4$ resonances, a broad resonance is observed in the ionization cross section [21] at energies near 16 $\mathrm{eV}$ indicating that it couples strongly to some of the autoionizing states in this energy region. Shpenik et al. [26] see large peaks in the excitation of $n=5,6{ }^{1} D_{2} \mathrm{Zn}^{*}$ states at 16.18 $\mathrm{eV}$. Interestingly, this feature does not appear in $\mathrm{n}=4$ excitation function, while in $n=5$ it appears to be two closely spaced resonances. The resonance appears also in the photon excitation function of the $4 s 4 p{ }^{1} P$ states as a dip located at $16.16 \mathrm{eV}$ with a convoluted width of $460 \mathrm{meV}$ [7] while lower resonances were not observed. The same $4 s 4 p{ }^{1} P$ excitation process is dominated by the $c$ resonance in the $\mathrm{n}=4$ resonance region.

\section{CONCLUSION}

The two complementary methods, angle differential elastic scattering and electron transmission, were used to study negative ion resonances in zinc. Data on elastic scattering below the first ionization threshold were compared with previous experimental and theoretical [9] resonance energies and to test assignments of individual resonances. Above the ionization threshold elastic scattering data indicate that the majority of resonances around $11 \mathrm{eV}$ occur in d partial wave consistent with the $3 d^{9} 4 s^{2} 4 p^{2}$ electron configuration. The assignments of symmetry are proposed based on comparison with isoelectronic autoionizing states of $\mathrm{Ga}$ atom and conservation of angular momentum and parity. The high sensitivity of the transmission experiments helped observe and establish precise energies of resonance features up to $16 \mathrm{eV}$.

Electron scattering via $3 d$ core excited negative ion resonances introduces a new complexity into the scattering process due to the close balance of different interatomic interactions and has not been modeled so far. To describe this scattering process, both the electron correlations arising from the opening of the $3 d$ core and sufficient coupling to the 
continuum have to be treated precisely. The calculations are very complex and computational requirements exceed presently available codes and computer support. However, the need for theoretical input is essential for full understanding of electron correlations which govern not only formation and decay of temporary negative ions but also the momentum coupling in zinc and other complex atoms.

\section{ACKNOWLEDGMENTS}

This work was supported by the Australian Research Council and the University of Western Australia. The technical assistance of Steve Key of UWA Mechanical workshop is acknowledged. P.D.B. acknowledges useful conversations with G. A. Gallup.
[1] S. J. Buckman and C. W. Clark, Rev. Mod. Phys. 66, 539 (1994).

[2] L. Pravica, D. Cvejanovic, J. F. Williams, and S. A. Napier, Phys. Rev. A 75, 030701(R) (2007).

[3] P. D. Burrow, J. A. Michejda, and J. Comer, J. Phys B: At., Mol. Opt. Phys. 9, 3225 (1976).

[4] J. P. Sullivan, P. D. Burrow, D. S. Newman, K. Bartschat, J. A. Michejeda, R. Panajotovic, M. Moghbelalhossein, R. P. McEachran, and S. J. Buckman, New J. Phys. 5, 159.1 (2003).

[5] O. B. Shpenik, I. P. Zapesochnyi, V. V. Sovter, E. E. Kontrosh, and A. N. Zavilopulo, Sov. Phys. JETP 38, 898 (1974).

[6] S. A. Napier, D. Cvejanovic, J. F. Williams, and L. Pravica, Phys. Rev. A 78, 022702 (2008).

[7] S. A. Napier, D. Cvejanovic, J. F. Williams, and L. Pravica, Phys. Rev. A 78, 032706 (2008).

[8] S. A. Napier, D. Cvejanovic, J. F. Williams, and L. Pravica, J. Phys. B 40, 1323 (2007).

[9] O. Zatsarinny and K. Bartschat, Phys. Rev. A 71, 022716 (2005).

[10] L. Sanche and G. J. Schulz, Phys. Rev. A 5, 1672 (1972).

[11] A. R. Johnston and P. D. Burrow, Phys. Rev. A 51, 406 (1995).

[12] A. Stamatovic and G. J. Schulz, Rev. Sci. Instrum. 41, 423 (1970).

[13] M. W. D. Mansfield, J. Phys B: At., Mol. Opt. Phys. 14, 2781 (1981).
[14] Y. Ralchenko, A. E. Kramida, and J. Reader, NIST Atomic Spectra Database (version 3.1.5), National Institute of Standards and Technology, Gaithersburg, MD, 2008 (http:// physics.nist.gov/asd3).

[15] C. G. Back, M. D. White, V. Pejcev, and K. J. Ross, J. Phys B: At., Mol. Opt. Phys. 14, 1497 (1981).

[16] H. G. Kuhn, Atomic Spectra (Longman Group Ltd., London, 1971).

[17] P. D. Burrow, J. A. Michejda, D. R. Lun, R. P. McEachran, D. S. Newman, and S. J. Buckman, J. Phys. B 31, L1009 (1998).

[18] D. S. Newman, M. Zubek, and G. C. King, J. Phys B: At., Mol. Opt. Phys. 18, 985 (1985).

[19] J. P. Connerade, Proc. R. Soc. London, Ser. A 354, 511 (1977).

[20] A. Hashizume and N. Wasada, Jpn. J. Appl. Phys. 18, 429 (1979).

[21] O. B. Shpenik, I. V. Chernishova, and J. E. Kontros, Radiat. Phys. Chem. 68, 277 (2003).

[22] K. Sommer, M. A. Baig, and J. Hormes, Z. Phys. D: At., Mol. Clusters 4, 313 (1987).

[23] G. Racah, Phys. Rev. 61, 537 (1942).

[24] D. H. Yu, P. A. Hayes, and J. F. Williams, J. Phys. B 30, L487 (1997).

[25] F. H. Read, J. N. H. Brunt, and G. C. King, J. Phys B: At., Mol. Opt. Phys. 9, 2209 (1976).

[26] O. B. Shpenik, M. M. Erdevdy, and J. E. Kontros, Radiat. Phys. Chem. 76, 587 (2007). 